\title{
Plants Disease Phenotyping using Quinary Patterns as Texture Descriptor
}

\author{
Wakeel Ahmad $^{1 *}$, S. M. Adnan Shah ${ }^{1}$ and Aun Irtaza ${ }^{1,2}$ \\ ${ }^{1}$ Department of Computer Science, \\ University of Engineering and Technology Taxila - PK \\ [e-mail: wakeel.ahmad@uettaxila.edu.pk] \\ [e-mail: syed.adnan@uettaxila.edu.pk] \\ ${ }^{2}$ Department of Computer and Electrical Engineering \\ University of Michigan-Dearborn, USA \\ [e-mail: aun.irtaza@uettaxila.edu.pk] \\ *Corresponding author: Wakeel Ahmad
}

Received March 10, 2020; revised May 8, 2020; accepted July 16, 2020;

published August 31, 2020

\begin{abstract}
Plant diseases are a significant yield and quality constraint for farmers around the world due to their severe impact on agricultural productivity. Such losses can have a substantial impact on the economy which causes a reduction in farmer's income and higher prices for consumers. Further, it may also result in a severe shortage of food ensuing violent hunger and starvation, especially, in less-developed countries where access to disease prevention methods is limited. This research presents an investigation of Directional Local Quinary Patterns (DLQP) as a feature descriptor for plants leaf disease detection and Support Vector Machine (SVM) as a classifier. The DLQP as a feature descriptor is specifically the first time being used for disease detection in horticulture. DLQP provides directional edge information attending the reference pixel with its neighboring pixel value by involving computation of their grey-level difference based on quinary value $(-2,-1,0,1,2)$ in $0^{\circ}, 45^{\circ}, 90^{\circ}$, and $135^{\circ}$ directions of selected window of plant leaf image. To assess the robustness of DLQP as a texture descriptor we used a research-oriented Plant Village dataset of Tomato plant (3,900 leaf images) comprising of 6 diseased classes, Potato plant (1,526 leaf images) and Apple plant (2,600 leaf images) comprising of 3 diseased classes. The accuracies of $95.6 \%, 96.2 \%$ and $97.8 \%$ for the above-mentioned crops, respectively, were achieved which are higher in comparison with classification on the same dataset using other standard feature descriptors like Local Binary Pattern (LBP) and Local Ternary Patterns (LTP). Further, the effectiveness of the proposed method is proven by comparing it with existing algorithms for plant disease phenotyping.
\end{abstract}

Keywords: Binary Patterns, Ternary Patterns, Directional Local Quinary Patterns, Classification, Early \& Late Blight, Tomato, Apple, and Potato Disease 


\section{Introduction}

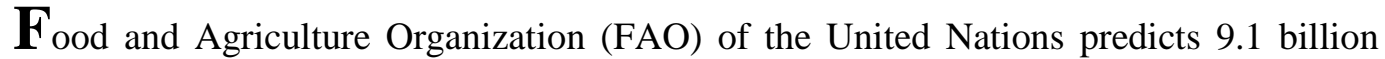
population on earth by 2050. Accordingly, the food production till 2050 must be increased by $70 \%$ [1]. Major challenges against increasing food production include: limited agricultural land, crops disease and availability of fresh water. Situation becomes worst due to climate changes that introduce various new types of plant disease, while our knowledge of physiological mechanism that links pathogen infection and presence of disease symptoms remains limited. In developing countries crops disease scouting is still done by visiting fields. It does not seem practically viable to consult an expert every time a plant is infected, secondly, neither farmer nor agriculture consultant can access every plant for problem inspection [2]. Detection of plants diseases thus urges researchers to develop and investigate computer vision-based techniques to ease this arduous task.

Considering major dietary source of the antioxidant lycopene and largest source of vegetables tomato and potato remain extremely important crop the world over. These antioxidants are enriched source of several vitamins and minerals including Vitamin C \& K1, Potassium and Folate (B9) [3]. Nutritional flavonoids are the major cause of decreased mortality, and apples are the main source of these flavonoids \& antioxidants. Only, in United States, in comparison with other fruits involve in antioxidant activities apples are the second highest since they provide $22 \%$ of phenolics compounds when compared to other fruits $[4,5]$. Further, consumption of fruits, vegetables and their related products has been linked to many health benefits regarding cancer prevention, heart disease and skin problems [6, 7]. According to a research, [8] fruits and vegetables have a significant shielding effect against a variety of cancer. Being major economic row crops, global sales from tomato, potato and apple exports in 2018 amounted to US\$21.43 billion [9]. Table 1 lists top countries that exported these crops of highest dollar value during 2018 [10]. Several yield-limiting factors can undermine the value of fruits and vegetables as agricultural commodity. These include abiotic factors like temperature, water, air, light and soil (nutrients) and biotic factors such as pests, insects, diseases and weeds. All these factors are potential threat to profitable production of fruits and vegetables. In this context, we have chosen tomato, potato, and apple as target crops for this study.

Table 1. List of Top Exporting Countries in 2018 for Tomato, Potato and Apple

\begin{tabular}{|c||l|c|c||l|l|l||l|c|c|}
\hline \multicolumn{1}{|c||}{} & \multicolumn{3}{c||}{ Tomato } & \multicolumn{3}{c|}{ Potato } & \multicolumn{3}{c|}{ Apple } \\
\hline \hline \multirow{2}{*}{ Rank } & Country & $\begin{array}{c}\text { Export } \\
\$ \text { bn }\end{array}$ & $\begin{array}{c}\% \\
\text { World } \\
\text { Total }\end{array}$ & Country & $\begin{array}{c}\text { Export } \\
\$ \text { \$bn }\end{array}$ & $\begin{array}{c}\text { \% } \\
\text { World } \\
\text { Total }\end{array}$ & Country & $\begin{array}{c}\text { Export } \\
\$ \text { bn }\end{array}$ & $\begin{array}{c}\% \text { World } \\
\text { Total }\end{array}$ \\
\hline 1 & Mexico & 2.300 & 23.7 & Netherland & 0.793 & 18.3 & China & 1.300 & 17.0 \\
2 & Netherland & 1.900 & 20.1 & France & 0.642 & 14.8 & USA & 1.000 & 13.3 \\
3 & Spain & 1.100 & 11.5 & Germany & 0.374 & 8.60 & Italy & 0.814 & 10.7 \\
4 & Morocco & 0.686 & 7.2 & China & 0.261 & 6.00 & Chile & 0.703 & 9.20 \\
5 & Canada & 0.411 & 4.3 & Canada & 0.252 & 5.80 & France & 0.573 & 7.50 \\
\hline
\end{tabular}


In this context, this study proposed an automatic and precise plant disease phenotyping system, which employ single channel grey level Directional Local Quinary Patterns (DLQP) [11] as a feature discriptor. Extraction of four directional quinary patterns (two uppers and two lowers) make the proposed approach rotation invariant. Further, due to considering magnitude of the intensity difference between the center reference pixel and neighboring ( $3 \times 3$ window) pixel the proposed method is extremely low imune to noise. Consequenlty, shown improved performance results over other similar local discriptors like Local Binary Pattern (LBP) [12] and Local Ternary Patterns (LTP) [13] for plant disease detection. An added advantage of the proposed method would be reduced computational complexity due to single channel grey level representation of the plant leaf image as a replacement of three color (R, G, and B) channels, details are given in materials and methods section. This paper is structured as follows: a brief review and related work is given in section 2 and in section 3 materials and methods are presented. Result discussion and evaluation of the proposed method are given in section 4 . Finally, section 5 derived the conclusion.

\section{Related Work}

Image processing techniques are applied to diagnosis of plant diseases in many crops such as wheat, maize, cotton, potato, tomato and apple etc. [14] proposed a pattern recognition-based system for identification and classification of disease infected cotton leaves. In this study researchers targeted the common diseases of cotton like Bacterial Blight, Myrothecium and Alternaria. Image segmentation was done using active contour model and Hus moments were extracted for feature extraction. The study claims 85\% accuracy. In [15] authors proposed an innovative cucumber's disease recognition method which is based on three sequential steps. First step is segmenting diseased image using K-means algorithm, second is extracting shape and color features from segmented images. The third and last step is to classify diseased images using Sparse Representation (SR). Study claims that SR reduced computations and improved the performance in terms of recognition. Proposed technique recognized seven cucumber diseases with $85.7 \%$ recognition rate.

The study [16] proposed a plant disease recognition method using leaf images. Initially diseased spot on leaf is segmented for extraction of feature vector. Then K-Nearest Neighbor (KNN) is applied to show proposed method's efficiency dealing with five kinds of maize diseases. Presented results justified the proposed technique for high disease recognition rates. [17] presents an extension of Neural Network (NN) model based on combination of three-color components titled as Three-Channel Convolutional Neural Networks (TCCNN). This approach has been successful in automatic extraction of high-level discriminant features from colored diseased leaf image resulted in higher efficiency in comparison with many cutting-edge systems of vegetable leaf disease recognition. In [18] authors present an image segmentation technique that can be used for automatic recognition and classification of infected plant leaves. The proposed technique was tested and verified on various fruits and vegetables including banana, beans, jackfruit, lemon, mango, potato, tomato, and sapota. Study claims less computational efforts with high efficiency. Accuracy rate of proposed model can be improved by using Artificial Neural Networks (ANN), Bayes Classifier, Fuzzy Logic and Hybrid algorithms.

In [19] author presented a Support Vector Machine (SVM) based approach for the detection and identification of unhealthy tomato leaves. Image processing technique has been major part of system using Gray-Level Co-occurrence Matrix (GLCM). In this study total 800 mages (healthy and infected) were used for training and testing while cross validation was done using 
$\mathrm{N}$-fold technique. High classification rate is reported using linear kernel function. In [20] an intensity-based feature extraction approach was presented for tomato plant disease recognition. During feature extraction phase, a total of ten intensity based statistical features were computed for each plant disease. These extracted features were used for classification using decision tree which generated $78 \%$ recognition accuracy rate. The study used images of six different types of plant disease infecting the leaves and stem.

Another study [21] presented a computer vision based system for detection of mycotic infections on tomato plant leaves. The proposed system is based on characterization of tomato leaves by Moving Picture Experts Group (MPEG) standard color descriptors which include Color Structure, Color Layout and Scalable Color Descriptors. The reported results show that calable Color Descriptors outperforms all other reported descriptors. In [22] author proposed a potato late blight prediction model using Support Vector Regression (SVR) named as BLITE-SVR. The study used 13 kinds of abiotic factors mainly including temperature, humidity and evaporation which showed very high correlation to the first date of occurrence of late blight on potato leaves. The proposed model claimed 64\% occurrence prediction.

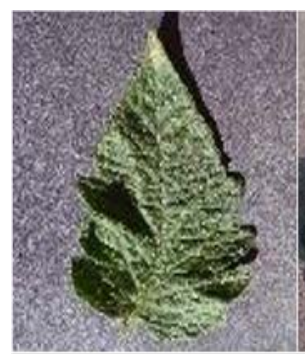

(a) Healthy

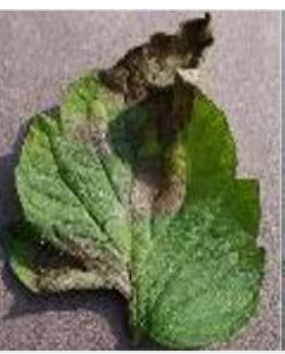

(b) Late Blight

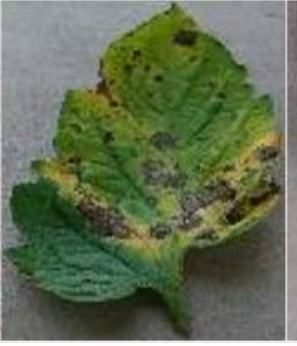

(c) Early Blight

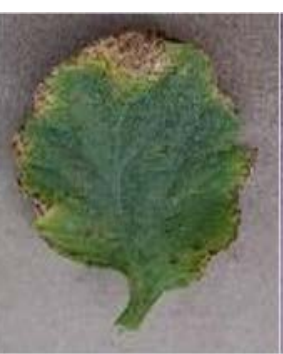

(d) Bacterial Spot

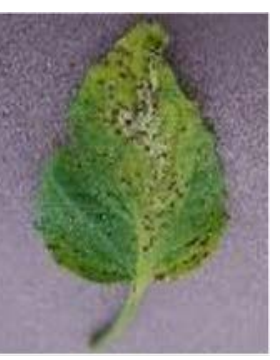

(e) Septoria

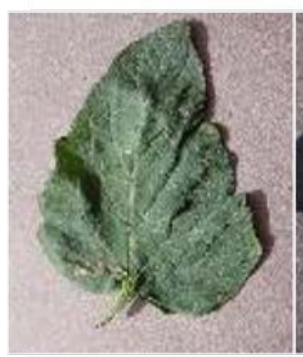

(f) Target Spot

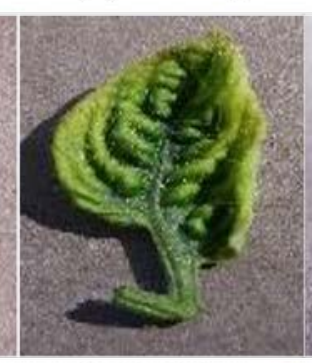

(g) YLCV

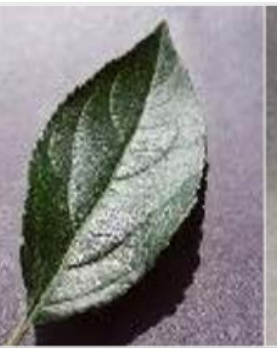

(h) Healthy

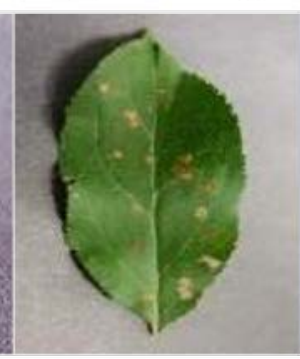

(i) Rust

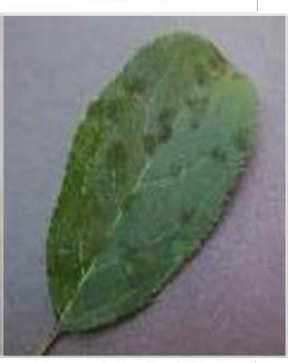

(j) SCAB

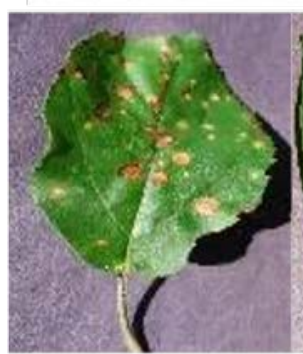

(k) Black Rot

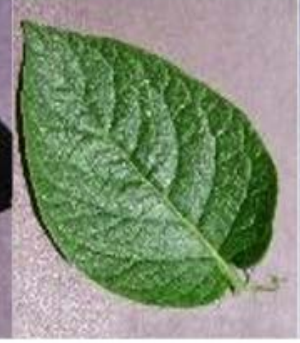

(1) Healthy

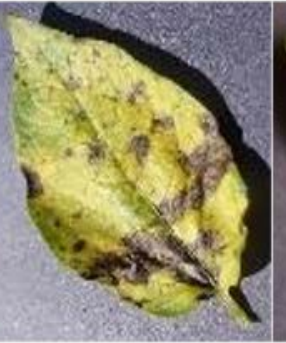

(m) Early Blight

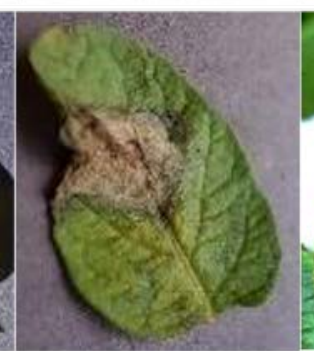

(n) Late Blight

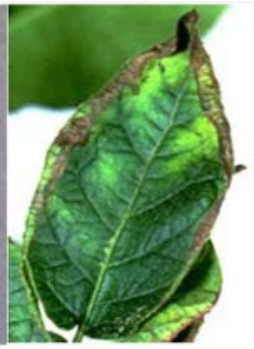

(o) Ring Rot

Fig. 1. Sample images from Plant Village dataset of Tomato [a-g], Apple [h-k], and Potato [l-o] 
Regardless of significant efforts, existing plant disease identification methods are not adequate or enough due to inherent discrepancies. It includes the detection which is not limited to: (1) the leaf image features extracted from diseased crops which are sensitive to illumination, direction and scaling of the images; (2) all selected features are playing important role in classification though they are just representing the algorithm strength. Further, it is difficult to determine which features are optimum and robust for disease recognition. To improve the accuracy and responsiveness of the diagnosis results, many techniques based on different features (i.e. color, texture, and shape) to classify healthy and diseased leaves have been proposed. Few of them have yielded good performance under controlled conditions whereas achieving accuracy in uncontrolled conditions remains a challenge. As far as the recently emerged Deep Learning (DL) techniques are concerend the image processing techniques are still prefered because of inadequacy in mechanism for learning abstractions through explicit verbal definitions. Moroever, DL techniques works best when there are thousands, millions or even billions of training examples [23]. Therefore, DL techniques are not quite fit yet for plant leaf disease classification due to lack of such infinite data and computational resources.

We used images from Plant-Village dataset published online at website www.Plant-Village.org [24], which includes images of several crops. To test and evaluate our proposed meathod, tomato, potato, and apple are choosen as the target crops because these are considered major economic row crops and secondly, due to high consumption rate in daily life. This study used 3900 tomato plant leaf images comprising of 6 diseased classes, 1,526 potato plant leaf images comprising of 3 disease classes and 2,600 apple leaf images comprising of 3 disease classes. Fig. 1. shows infected leaf images of target crops.

\section{Materials and methods}

\subsection{Local Binary Patterns}

Local Binary Pattern (LBP) [12] is a texture-based pattern operator. Efficiency with simplicity is main characteristic of LBP. The main advantage offered by LBP is to label neighboring pixel values with reference to their center pixel from selected window and computing result as binary numbers. LBP has been applied to diverse applications, more specifically dealing in face detection. Main contribution of local binary patterns as an operator has been the robustness to monotonic gray-scale changes like illumination variations. LBP represents the window's centered reference pixel $n_{c}$ and $n_{p}$ neighbor's gray values which are the total neighbors $(\mathrm{N})$ of that window and its radius $R$ of the neighborhood as shown in Eq. (1). Here value of function $f_{1}$ depends upon $X$ as defined by Eq. (2).

$$
\begin{aligned}
& L B P_{Y, R}=\sum_{Y=1}^{N} 2^{Y-1} * f_{1}\left(n_{p}-n_{C}\right) \\
& f_{1}(x)= \begin{cases}1 & x \geq 0 \\
0 & \text { otherwise }\end{cases}
\end{aligned}
$$

LBP in deals with texture classification as a discriminative feature descriptor and shows its invariance for monotonic gray level transformation and high resistance to lighting effects. LBP is extremely noise sensitive due to center reference pixel thresholding for its neighbor gray levels as shown in Fig. 2. 


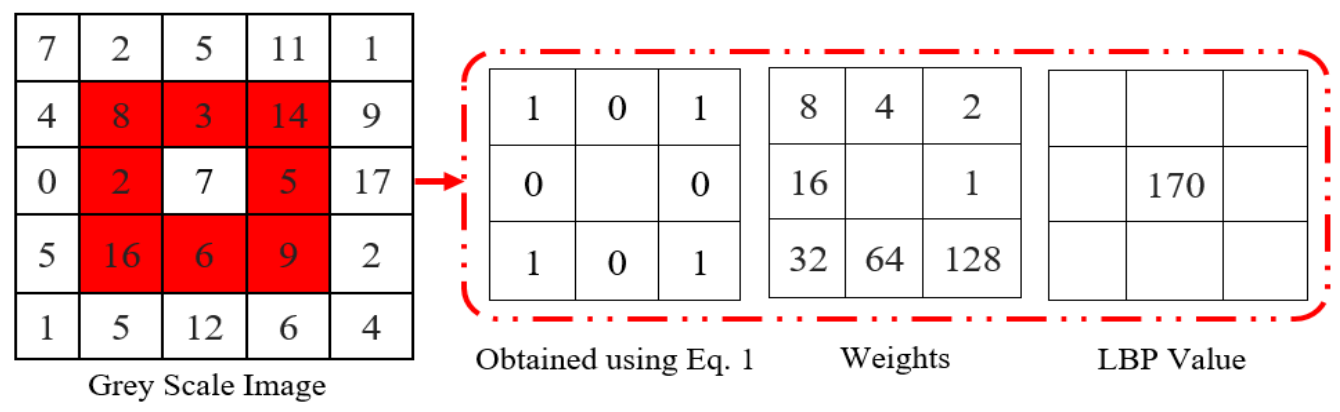

Fig. 2. Calculation of LBP value from its neighbourhood

\subsection{Local Ternary Patterns}

Local ternary pattern (LTP) [13] as a feature descriptor uses 3-valued code, gray-levels in region with range of width $\pm r_{t}$ around $p_{c}$ central pixel value are quantized to zero, ones above this are quantized to +1 and ones below it to -1 , i.e., the indicator $S^{\prime}(g)$ is replaced with a 3-valued function. Here $r_{t}$ is threshold specified by the user so LTP codes are more resistant to noise. As defined by Eq. (3).

$$
S^{\prime}\left(g, p_{c}, r_{t}\right)=\left\{\begin{array}{cl}
1, & g \geq p_{c}+r_{t} \\
0, & \left|g-p_{c}\right|<r_{t} \\
-1, & g \leq p_{c}-r_{t}
\end{array}\right.
$$

Where $g=\left(p_{i}-p_{c}\right)$; here $p_{i}$ is the neighboring pixel values, and $p_{c}$ central pixel value

\subsection{Directional Local Quinary Pattern (DLQP)}

DLQP [11] features have been computed for single channel gray scale images and Directional Binary Code (DBC) is used to encode directional edge information [25]. For a gray scale image $\left(I_{m g}\right)$, first-order derivatives $\left(I_{m g}^{\prime}\left(d_{k}\right)\right)$, occur first, along $0^{\circ}, 45^{\circ}, 90^{\circ}$, and $135^{\circ}$ directions. Detailed calculations are shown in Fig. 3. For $\left(I_{m g}\right)$, first order derivatives are calculated for each direction used in Eq. (4), here $d$ denotes the direction $\left(0^{\circ}, 45^{\circ}, 90^{\circ}\right.$, and $135^{\circ}$ ) and $d_{c}$ is the intensity of the center pixel in a respective direction. The local quinary values are calculated using Eq. (5), where directional edges are calculated using Eq. (6).

$$
\begin{aligned}
& I_{m g}^{\prime}\left(d_{k}\right)=I_{m g}\left(d_{c}\right)-I_{m g}\left(d_{k}\right) ; \quad k=1,2,3,4 \\
& f_{2}\left(p_{c}, \tau_{2}, \tau_{1}\right)=\left\{\begin{array}{ll}
+2, & p_{c} \geq \tau_{2} \\
+1, & \tau_{1} \leq p_{c}>\tau_{2} \\
0, & -\tau_{1}>p_{c}<\tau_{1} \\
-1, & \tau_{2}<p_{c} \leq-\tau_{1} \\
-2, & p_{c} \leq-\tau_{2}
\end{array} \mid\right.
\end{aligned}
$$

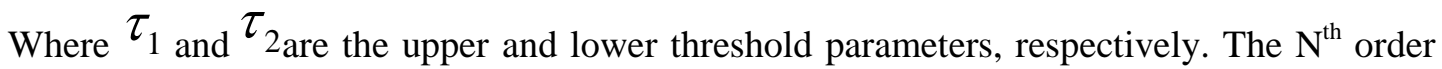
DLQP for Eq. (6) is defined using Eq. (7). 


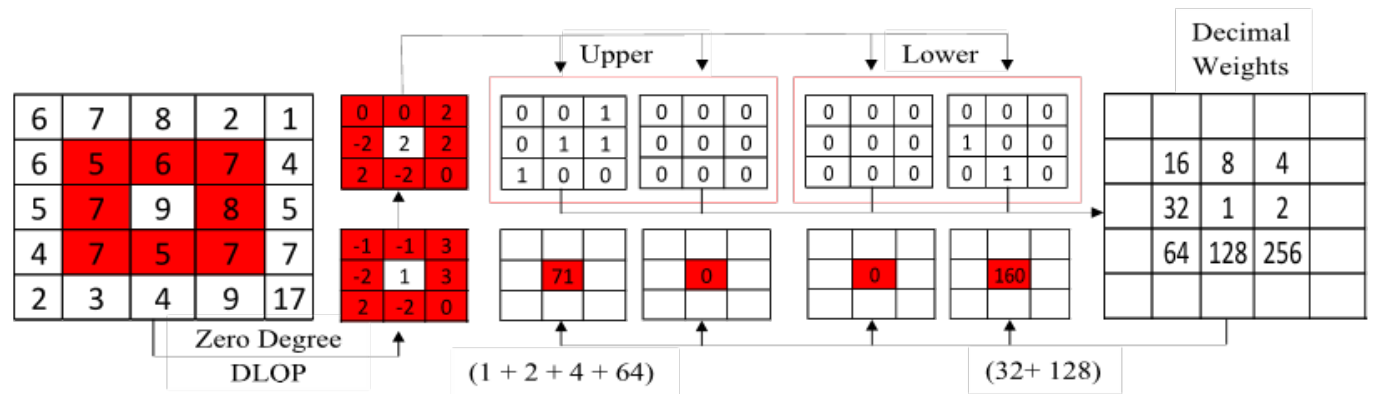

Fig. 3. Calculation of Zero Degree DLQP

$$
\begin{aligned}
& \hat{I}_{\alpha}^{D L Q P}\left(d_{c}\right)=f_{3}\left(I_{m g}^{\prime}\left(d_{l}\right)\right) ; l=(1+\alpha / 45) " \alpha=0^{0}, 45^{0}, 90^{0} \text { and } 135^{0}
\end{aligned}
$$

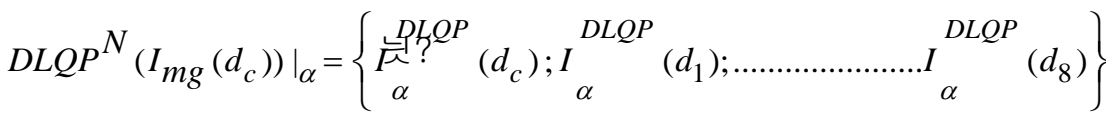

Complete description of DLQPs is as follows. Steps for the calculation of quinary pattern in 0 degree are explained in Fig. 3. After applying the 0-degree first order derivative we retrieved $-1,-1,3,-2,1,3,2,-2,0$ representing texture information. Further, derivatives are coded into quinary pattern $0,0,2,-2,2,2,2,-2,0$ using upper and lower thresholds ( $\tau_{1}=2$ and $\tau_{2}=1$ ). These patterns are further coded in two upper and two lower binary patterns. First upper pattern is attained by replacing 0 for $0,1,-1,-2$ and retaining 2 by 1 . Similarly, other upper pattern is attained by retaining 1 by 1 and replacing 0 for other values in said pattern. Likewise, same procedure is applied to the two lower patterns by retaining -1 and -2 by 1 accordingly. Now quinary pattern was successfully converted into four binary patterns, two upper and two lower. Finally, weights are applied to each pattern to get four decimal numbers pointed to DLQP. Constructing DLQP histogram for each pattern using 20 bins and shaping it in final feature vector ready to be used by SVM for classification. The obtained patterns for each directions is shown in Fig. 4, where Fig. 5 illustrates a complete flow diagram of the proposed system.

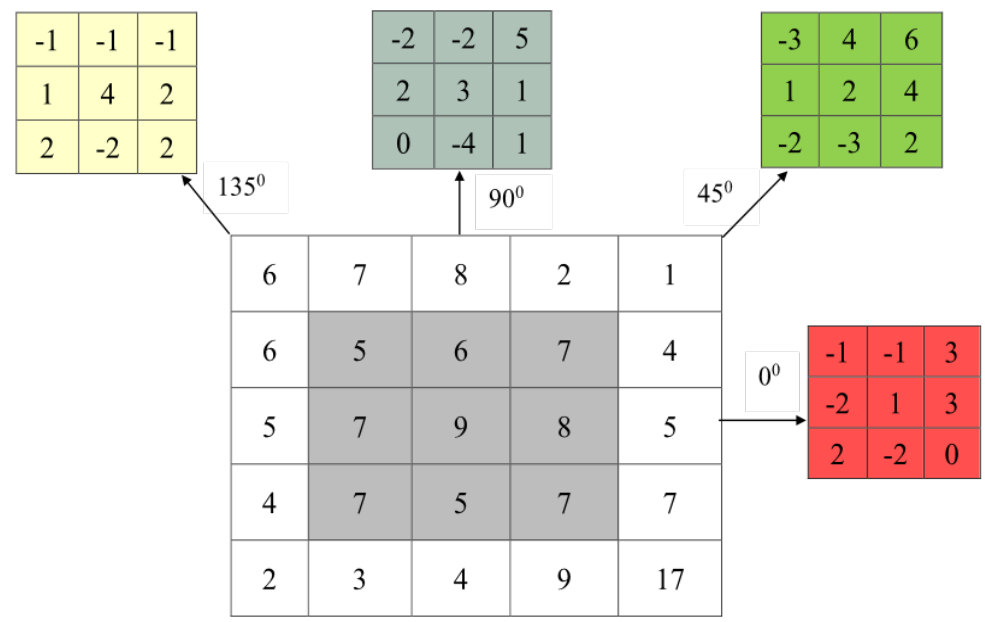

Fig. 4. DLQP patterns for each directions 


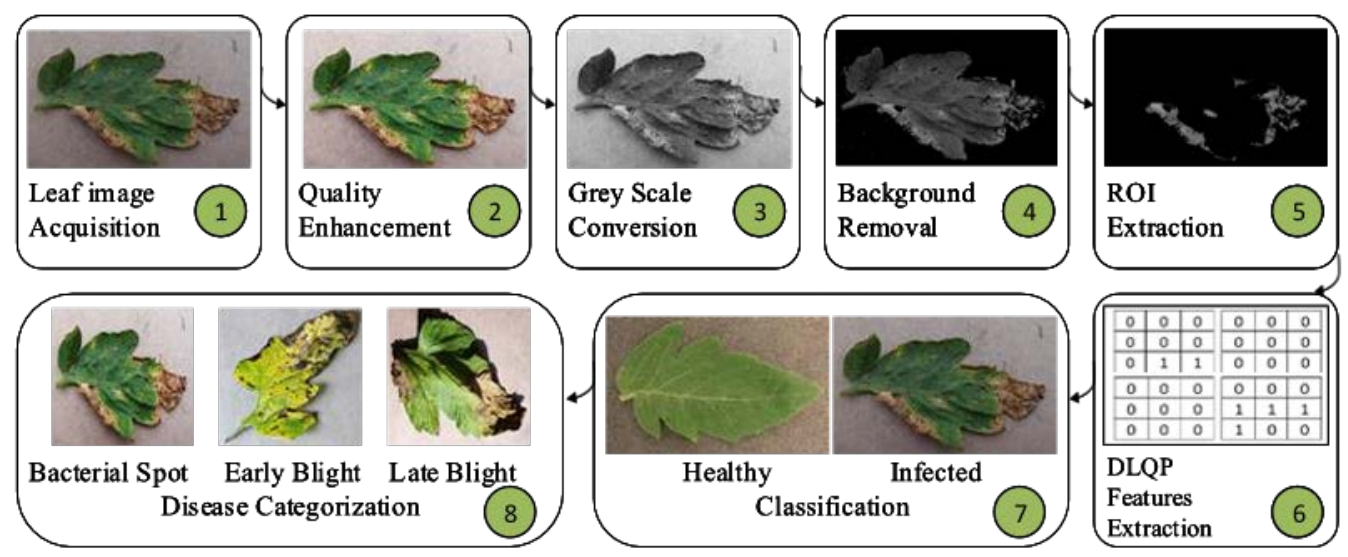

Fig. 5. Flow diagram of Proposed System

Dataset images are converted to grey scale (having value from 0 to 255) with dimension of 256 x 256 pixels each. Quality of the image is enhanced using histogram equalization technique as preprocessing step. Subsequently, the background of image is removed, and Region of Interest (ROI) is extracted by segmenting and targeting the diseased portion. Now the system is ready to extract features on this image by applying DLQP descriptor. After getting the final feature vector and in classification phase all three categories, namely tomato, potato, and apple, are grouped in different disease classes. In case of tomato, classes are Bacterial Spot, Early Blight, Late Blight, Septoria, Target Spot and YLCV. For potato, classes are Early Blight, Late Blight and Ring Rot, and for apple SCAB, Cedar Rust and Black Rot are taken. Dataset images were in JPEG format and converted into grayscale files.

\section{Results and Discussion}

Performance of the proposed system is evaluated in terms of Accuracy, Precision, Recall, F-1 Score and Error Rate. Here, specificity as precision rate determines the ability for correct classification of plant leaf disease against total number of detected disease classes. Precision can be calculated using Eq. (8), the capability of the disease phenotyping system for the association of model to its concerned class is computed using Eq. (9). To evaluate the accuracy of classification performed by the proposed technique F-1 Score is also computed, which imparts the balance or weighted average representation between the precision and the recall. F-1 Score is calculated using Eq. (10). Another performance metric adopted for the performance assessment is the accuracy measure, and is calculated using Eq. (11). Error rate is also calculated using Eq. (12).

$$
\begin{aligned}
& \text { Precision Rate }=\frac{\text { True Positive }}{\text { True Positive }+ \text { False Positive }} \\
& \text { Recall Rate }=\frac{\text { True Positive }}{\text { True Positive }+ \text { False Negative }} \\
& F-1 \text { Score }=2 * \frac{(\text { Precision } * \text { Recall })}{\text { Precision }+ \text { Recall }}
\end{aligned}
$$




$$
\begin{aligned}
& \text { Accuracy Rate }=\frac{\text { True Positive }+ \text { True Negative }}{\text { Total Positive }+ \text { Total Negative }} \\
& \text { Error Rate }=\frac{\text { False Positive }+ \text { False Negative }}{\text { Total Positive }+ \text { Total Negative }}
\end{aligned}
$$

For disease classification Support Vector Machine (SVM) is used as a classifier. After getting the training set, the random selection of $70 \%$ of the data for training and $30 \%$ for testing purposes has been carried out. Fig. 6 reflects the comparison results for accuracies, where DLQP achieve $95.6 \%$ for Tomato Plants while $97.8 \%$ on Apple and $96.2 \%$ on Potato plants using the Medium Gaussian kernel for SVM.

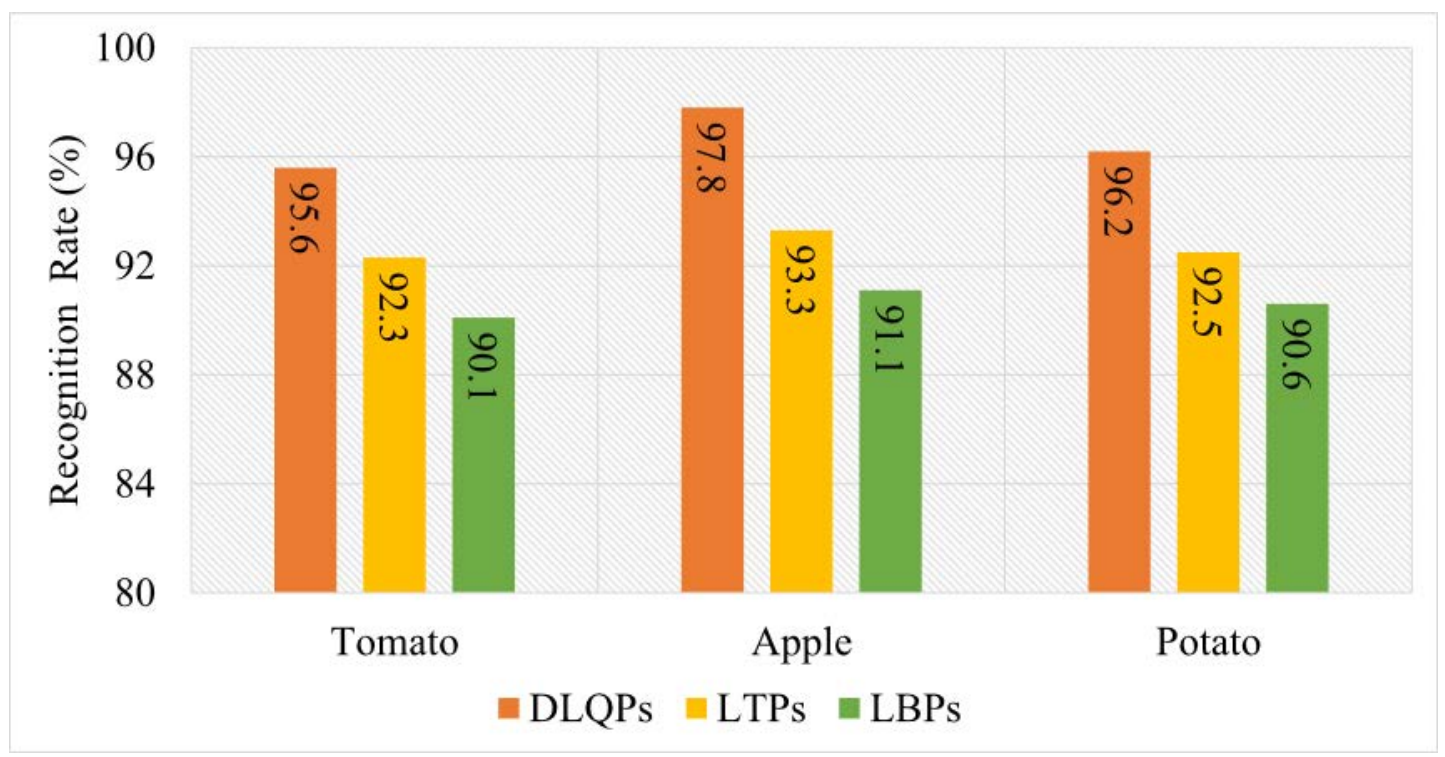

Fig. 6. Recognition rates using different local patterns

To reveal the capabilities of DLQP as a reliable image descriptor, system is evaluated by performing its validation over multi-category problem. Classification results over the multiclass problem confirm the robustness of DLQPs on plant disease phenotyping as the best feature descriptor. The high correlation between the classes makes the instance recognition problem even more challenging. Due to high correlation, miss-association rate is high as it increases classification biasness due to smaller instances in skewed class. The diversity of content in training samples also favors incorrect associations. Class imbalance raises a series of issues like class overlapping and small disjuncts that further increase the difficulty of classifier's learning. However, optimal feature basis increases feature separability amongst classes and, hence, ensures improved classification results. Precision graph for different diseases of apple are shown in Fig. 7, where precision graph for potato and tomato disease classes are shown using Fig. 8 and Fig. 9 respectivly. 


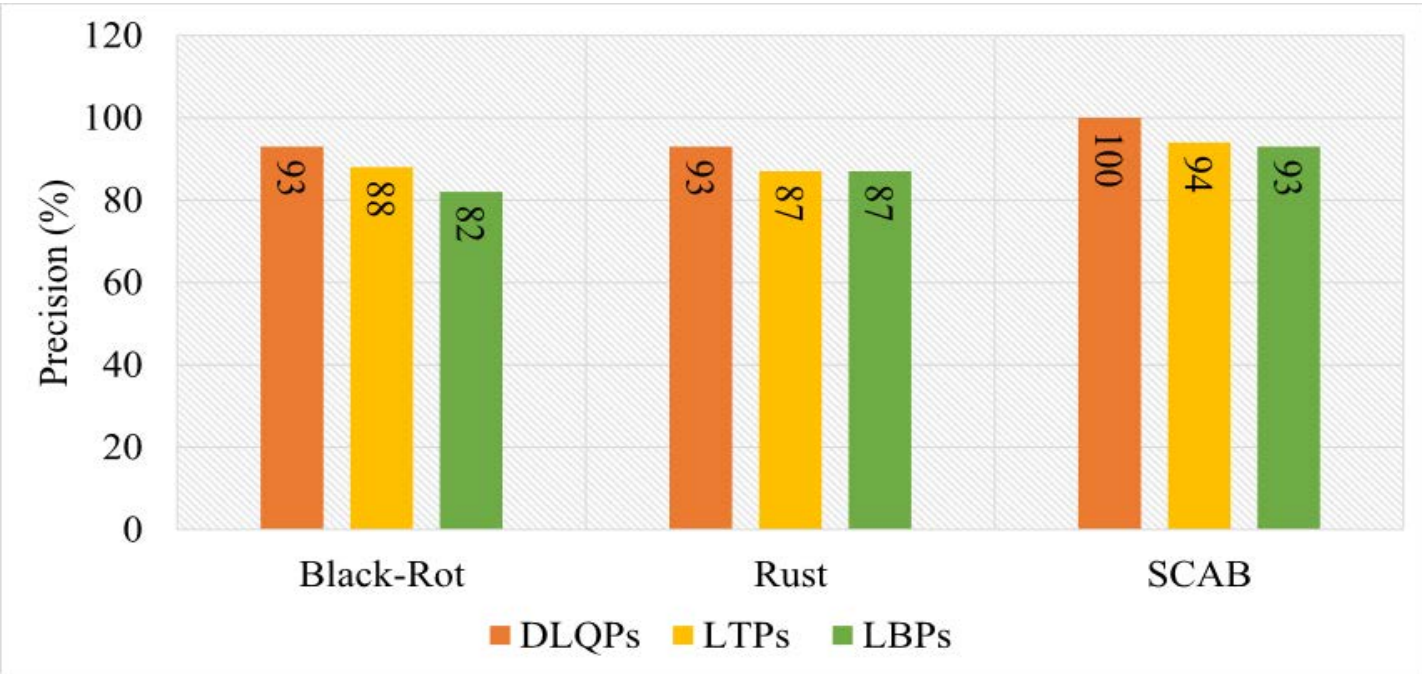

Fig. 7. Precision Graphs for Apple Disease Classes

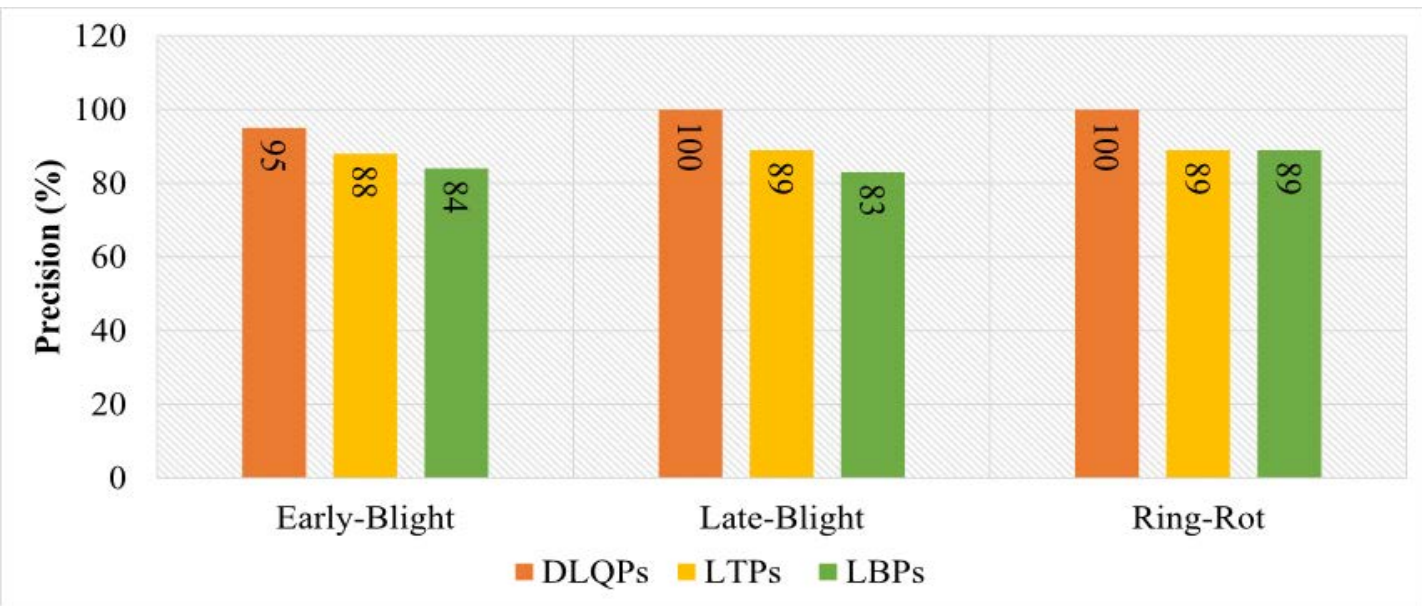

Fig. 8. Precision Graphs for Potato Disease Classes

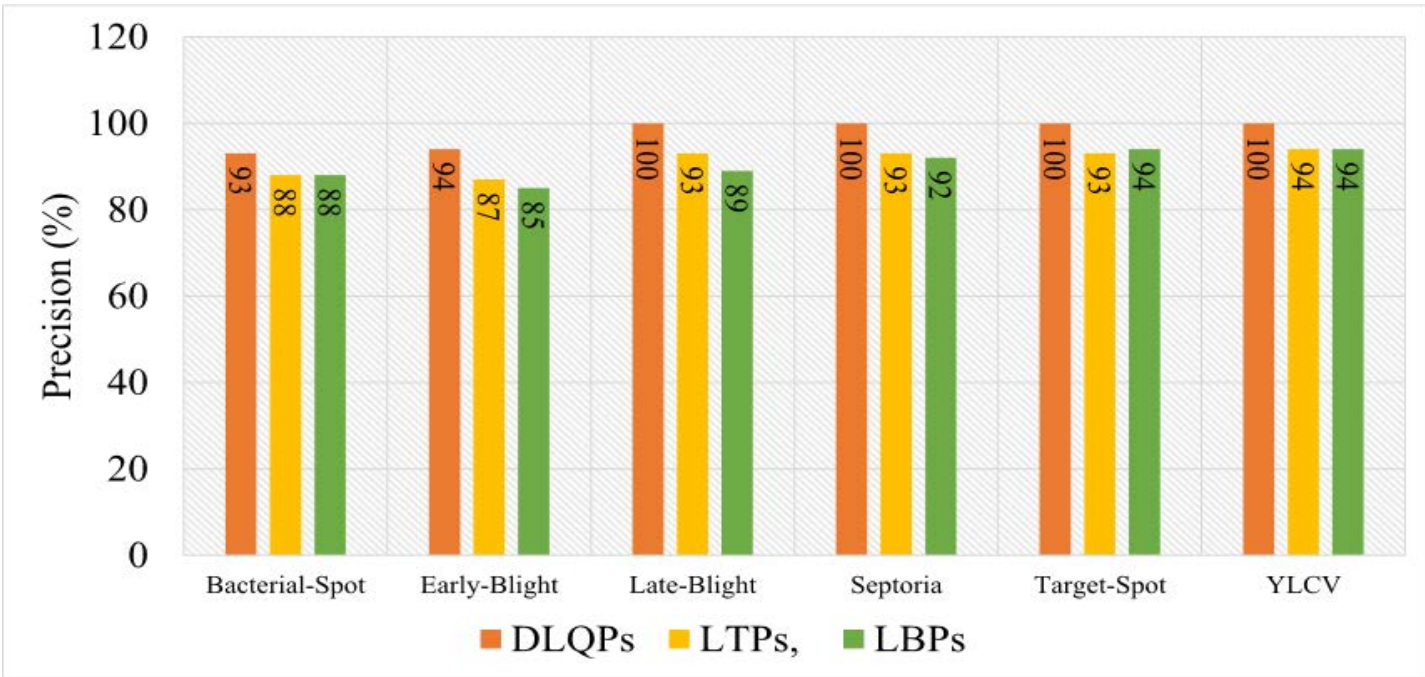

Fig. 9. Precision Graphs for Tomato Disease Classes 
Table 2. Multiclass Evaluation Results for Tomato, Potato and Apple Disease Classes

\begin{tabular}{|c|c|c|c|c|c|c|c|c|c|c|}
\hline \multirow{2}{*}{ ô้ } & \multirow{2}{*}{ 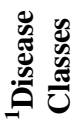 } & \multicolumn{3}{|c|}{ Recall } & \multicolumn{3}{|c|}{ F-1 Score } & \multicolumn{3}{|c|}{ False Discovery Rate } \\
\hline & & DLQPs & LTPs & LBPs & DLQPs & LTPs & LBPs & DLQPs & LTPs & LBPs \\
\hline \multirow{6}{*}{ 苞 } & BS & 0.93 & 0.94 & 0.93 & 0.93 & 0.94 & 0.88 & 0.07 & 0.06 & 0.18 \\
\hline & $\mathrm{EB}$ & 1.00 & 0.87 & 0.73 & 0.91 & 0.87 & 0.76 & 0.17 & 0.13 & 0.21 \\
\hline & LB & 1.00 & 0.93 & 1.00 & 1.00 & 0.93 & 0.94 & 0.00 & 0.07 & 0.11 \\
\hline & SP & 0.94 & 0.87 & 0.73 & 0.97 & 0.87 & 0.85 & 0.00 & 0.13 & 0.00 \\
\hline & TS & 0.87 & 0.93 & 1.00 & 0.93 & 0.93 & 0.97 & 0.00 & 0.07 & 0.06 \\
\hline & YL & 1.00 & 1.00 & 1.00 & 1.00 & 1.00 & 1.00 & 0.00 & 0.00 & 0.00 \\
\hline \multirow{3}{*}{$\frac{0}{2}$} & $\mathrm{BR}$ & 0.93 & 0.93 & 0.93 & 0.93 & 0.90 & 0.88 & 0.07 & 0.13 & 0.18 \\
\hline & CR & 0.93 & 0.87 & 0.87 & 0.93 & 0.93 & 0.93 & 0.07 & 0.00 & 0.00 \\
\hline & SC & 1.00 & 1.00 & 0.93 & 1.00 & 0.97 & 0.93 & 0.00 & 0.06 & 0.07 \\
\hline \multirow{3}{*}{$\begin{array}{l}\stackrel{0}{\overparen{Z}} \\
\stackrel{0}{0} \\
0\end{array}$} & EB & 1.00 & 0.83 & 0.89 & 0.95 & 0.91 & 0.86 & 0.10 & 0.00 & 0.16 \\
\hline & LB & 0.89 & 0.94 & 0.83 & 0.94 & 0.92 & 0.91 & 0.00 & 0.11 & 0.00 \\
\hline & $\mathrm{RR}$ & 1.00 & 1.00 & 1.00 & 1.00 & 0.94 & 0.94 & 0.00 & 0.11 & 0.11 \\
\hline
\end{tabular}

F1-score, recall, and false discovery rates are listed in Table 2. Results for selected evaluation parameters against relative schemes clearly signify the reliability of DLQP as feature descriptor. ROC curves using DLQP based features on Tomato images is shown in Fig. 10, while for Potato and Apple images ROC is shown in Fig. 11 and Fig. 12 resepctivly.

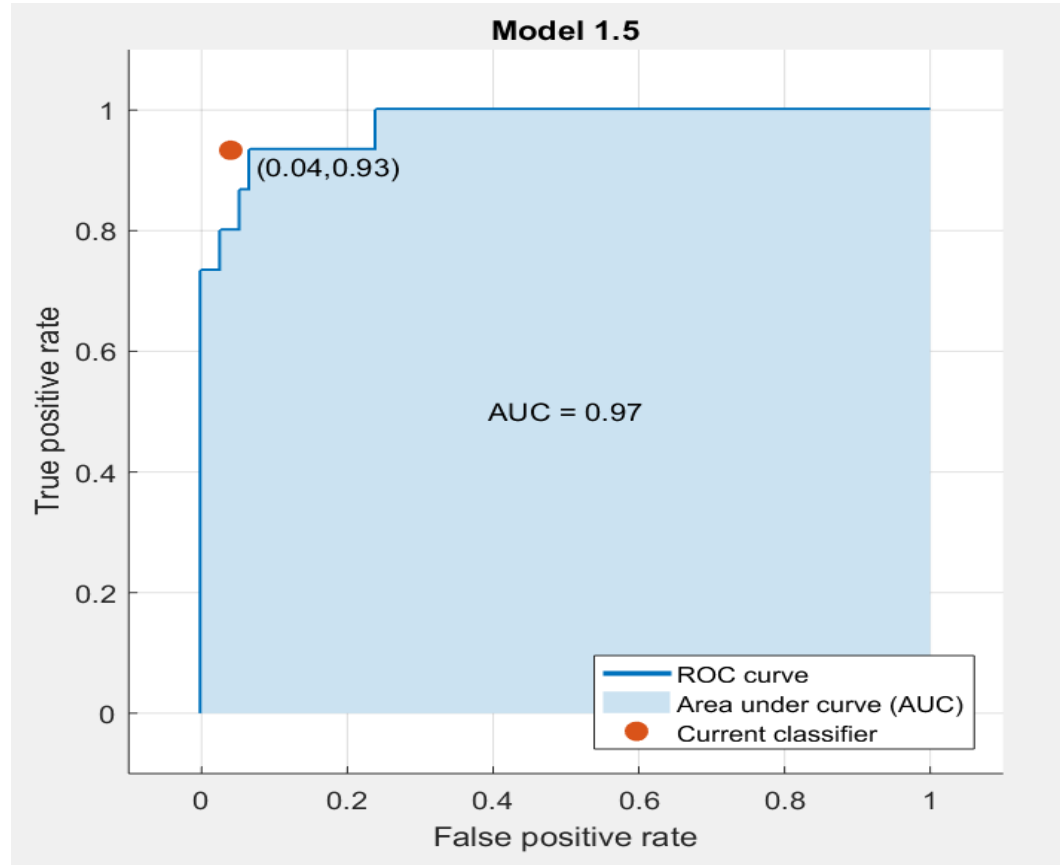

Fig. 10. RoC Curve using DLQPs based Features on Tomato images

\footnotetext{
${ }^{1}$ Bacterial Spot $=$ BS, Early Blight $=$ EB, Late Blight $=$ LB, Septoria $=$ SP, Target Spot $=$ TS and YLCV $=$ YL, Ring Rot $=$ RR, SCAB $=$ SC, Cedar Rust $=$ CR and Black Rot $=$ BR
} 


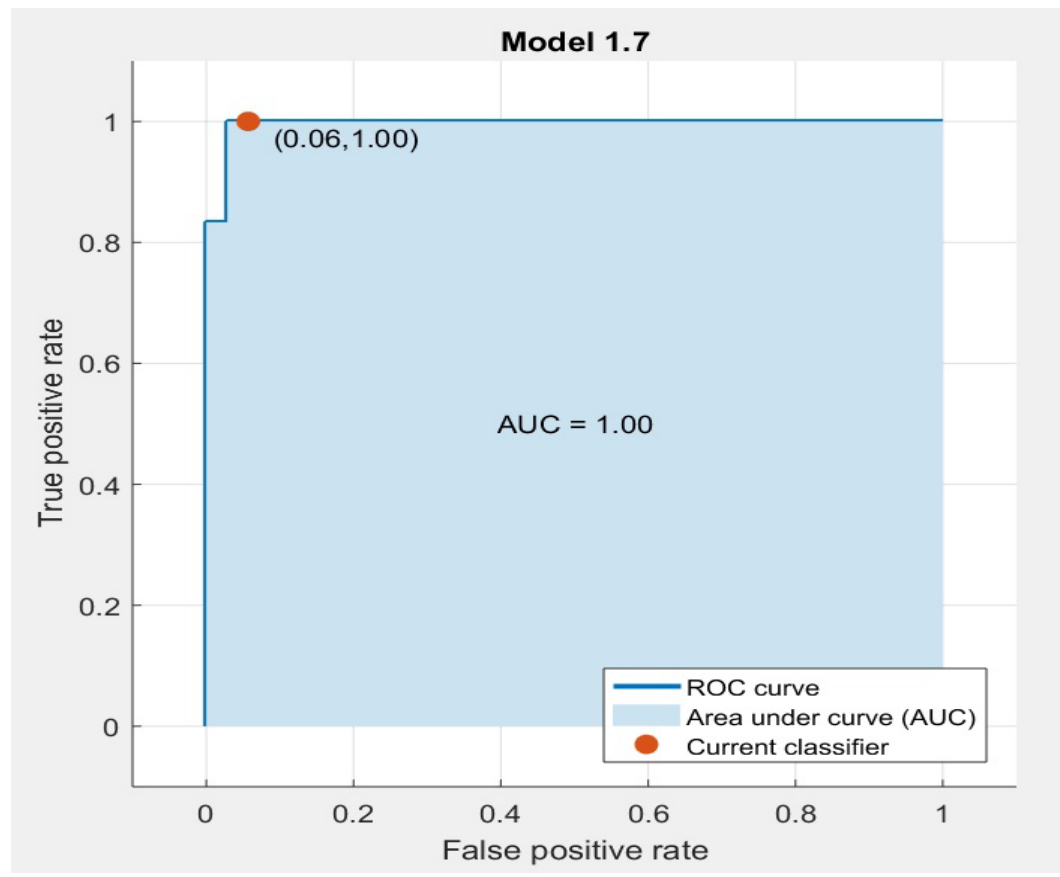

Fig. 11. RoC Curve using DLQPs based Features on Potato images

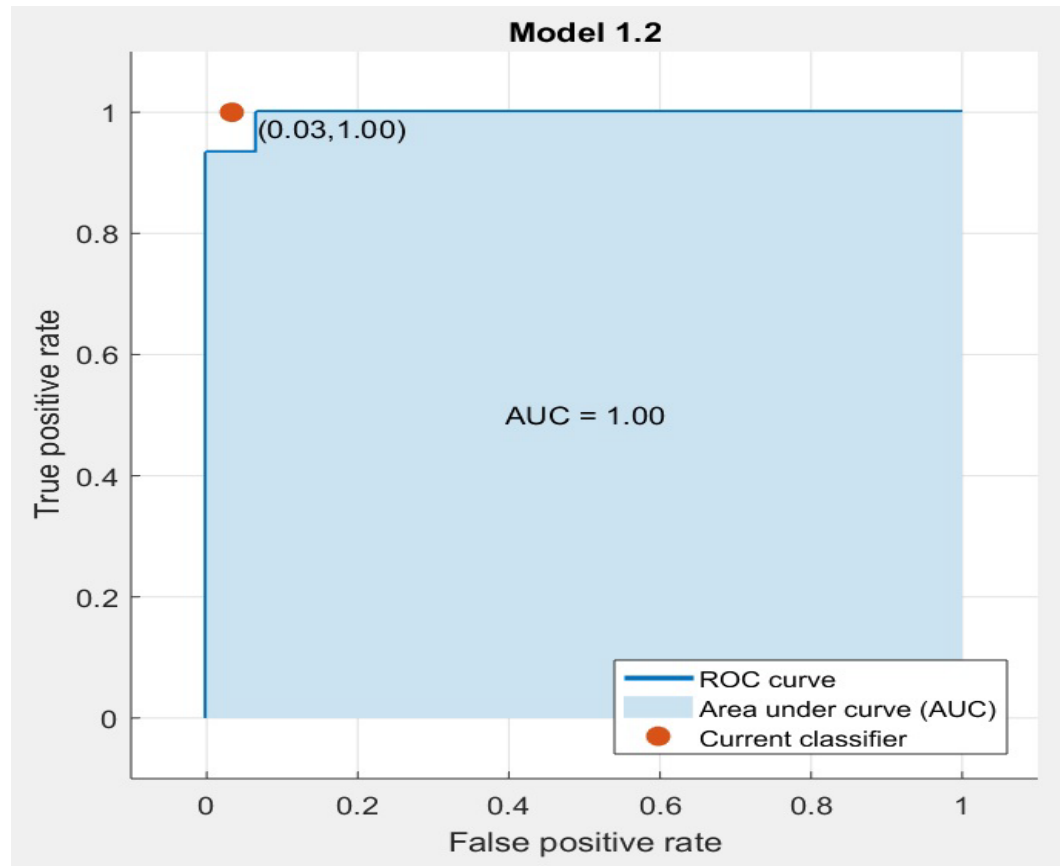

Fig. 12. RoC Curve using DLQPs based Features on Apple images 
Table 3. Multiclass Evaluation Results for Tomato, Potato and Apple Disease Classes

\begin{tabular}{|c||c||c||c|}
\hline Ref & Year & Techniques Used & Avg. Accuracy\% \\
\hline$[15]$ & 2017 & K-means clustering with Sparse Representation & 85.7 \\
{$[26]$} & 2017 & Deep CNN & 95.0 \\
{$[27]$} & 2018 & Canny Edge Detection and Mahalanobis Distance & 96.0 \\
{$[28]$} & 2018 & K-means clustering with SVM & 96.0 \\
{$[29]$} & 2018 & Fast K-means Clustering with SVM & 84.0 \\
{$[30]$} & 2018 & PCA, Entropy, \& Skewness with SVM & 92.1 \\
{$[2]$} & 2019 & Local Binary Patterns with One Class Classification & 95.0 \\
{$[31]$} & 2019 & SVM & 89.6 \\
{$[32]$} & 2020 & Deep Neural Network & 87.7 \\
{$[33]$} & 2020 & Radial Basis Function Neural Network & 95.0 \\
Proposed & ---- & Directional Local Quinary Pattern with SVM & $\mathbf{9 6 . 5}$ \\
\hline
\end{tabular}

Comparison of proposed technique with existing techniques of crop diseases identification is presented in Table 3. It can be witnessed that DLQP as feature descriptor outperformed other descriptors, including LTP and LBP, by achieving highest accuracies.

\section{Conclusion}

This study proposed an automatic approach for image-based phenotyping of plant disease using Directional Local Quinary Patterns (DLQP) as feature descriptor and Support Vector Machine (SVM) as a classifier. The proposed DLQP based system is specifically used for agricultural applications. Six tomato leaf diseases, three potato, and three apple leaf diseases are taken for experimentation. For each disease we performed a classification process and compared the individual performance of DLQP, LTP and LBP as feature descriptors. It is found that proposed DLQP texture feature descriptor improves the performance for plant disease phenotyping. The maximum detection efficiencies of $97.8 \%$ for apple, $95.6 \%$ for tomato, and $96.2 \%$ for potato are achieved using DLQP and Medium Gaussian kernel for SVM. Also, a comprehensive comparison shows that the proposed method performs significantly well as compared to existing methods. The proposed system provides promising results for plant disease phenotyping but there is a scope for improvement by using combination of other shape and color-based feature descriptors with DLQP. Moreover, use of ensemble methods as classifier may improve the results.

\section{Acknowledgment}

We are thankful to Plant-Village for sharing their research-oriented dataset with us. Contribution of authors are as follows, Wakeel Ahmad did the experiments and wrote the paper with Syed M. Adnan, Aun Irtaza interpreted the results. 


\section{References}

[1] J. Bruinsma, "The resource outlook to 2050: by how much do land, water and crop yields need to increase by 2050," Expert meeting on how to feed the world in, pp. 24-26, 2009.

[2] X. E. Pantazi, D. Moshou, and A. A. Tamouridou, "Automated leaf disease detection in different crop species through image features analysis and One Class Classifiers," Computers and electronics in agriculture, vol. 156, pp. 96-104, 2019. Article (CrossRef Link)

[3] L. D'Elia, G. Barba, F. P. Cappuccio, and P. Strazzullo, "Potassium intake, stroke, and cardiovascular disease: a meta-analysis of prospective studies," Journal of the American College of Cardiology, vol. 57, no. 10, pp. 1210-1219, 2011. Article (CrossRef Link)

[4] J. Sun, Y.-F. Chu, X. Wu, and R. H. Liu, "Antioxidant and antiproliferative activities of common fruits," Journal of agricultural and food chemistry, vol. 50, no. 25, pp. 7449-7454, 2002. Article (CrossRef Link)

[5] J. A. Vinson, X. Su, L. Zubik, and P. Bose, "Phenol antioxidant quantity and quality in foods: fruits," Journal of agricultural and food chemistry, vol. 49, no. 11, pp. 5315-5321, 2001. Article (CrossRef Link)

[6] J. Karppi, J. A. Laukkanen, T. H. Mäkikallio, and S. Kurl, "Low serum lycopene and $\beta$-carotene increase risk of acute myocardial infarction in men," The European Journal of Public Health, vol. 22, no. 6, pp. 835-840, 2012. Article (CrossRef Link)

[7] J. Karppi, J. A. Laukkanen, J. Sivenius, K. Ronkainen, and S. Kurl, "Serum lycopene decreases the risk of stroke in men: a population-based follow-up study," Neurology, vol. 79, no. 15, pp. 1540-1547, Oct 9 2012. Article (CrossRef Link)

[8] G. Block, B. Patterson, and A. Subar, "Fruit, vegetables, and cancer prevention: a review of the epidemiological evidence," Nutrition and cancer, vol. 18, no. 1, pp. 1-29, 1992. Article (CrossRef Link)

[9] World's Top Exports (WTEx), WTEx is an independent educational website designed to inspire researchers around the globe. Available: http://www.worldstopexports.com/, December 18, 2019.

[10] Central Intelligence Agency, The World Factbook, Field Listing: exports commodities Available: https://www.cia.gov/library/publications/the-world-factbook/, November 10, 2019.

[11] S. K. Vipparthi and S. K. Nagar, "Color directional local quinary patterns for content based indexing and retrieval," Human-centric Computing and Information Sciences, vol. 4, no. 1, pp. 1-13, 2014. Article (CrossRef Link)

[12] T. Ojala, M. Pietikäinen, and D. Harwood, "A comparative study of texture measures with classification based on featured distributions," Pattern recognition, vol. 29, no. 1, pp. 51-59, 1996. Article (CrossRef Link)

[13] X. Tan and B. Triggs, "Enhanced local texture feature sets for face recognition under difficult lighting conditions," IEEE transactions on image processing, vol. 19, no. 6, pp. 1635-1650, 2010. Article (CrossRef Link)

[14] P. Rothe and R. Kshirsagar, "Cotton leaf disease identification using pattern recognition techniques," in Proc. of 2015 International Conference on Pervasive Computing (ICPC), pp. 1-6, 2015. Article (CrossRef Link)

[15] S. Zhang, X. Wu, Z. You, and L. Zhang, "Leaf image based cucumber disease recognition using sparse representation classification," Computers and electronics in agriculture, vol. 134, pp. 135-141, 2017. Article (CrossRef Link)

[16] S. Zhang, Y. Shang, and L. Wang, "Plant disease recognition based on plant leaf image," J. Anim. Plant Sci, vol. 25, no. 3, pp. 42-45, 2015.

[17] S. Zhang, W. Huang, and C. Zhang, "Three-channel convolutional neural networks for vegetable leaf disease recognition," Cognitive Systems Research, vol. 53, pp. 31-41, 2019. Article (CrossRef Link)

[18] V. Singh and A. K. Misra, "Detection of plant leaf diseases using image segmentation and soft computing techniques," Information processing in Agriculture, vol. 4, no. 1, pp. 41-49, 2017. Article (CrossRef Link) 
[19] U. Mokhtar, N. El Bendary, A. E. Hassenian, E. Emary, M. A. Mahmoud, H. Hefny, and M. F. Tolba, "SVM-based detection of tomato leaves diseases," in Proc. of Intelligent Systems' 2014, ed: Springer, pp. 641-652, 2015. Article (CrossRef Link)

[20] H. Sabrol and S. Kumar, "Intensity based feature extraction for tomato plant disease recognition by classification using decision tree," International Journal of Computer Science and Information Security, vol. 14, no. 9, p. 622, 2016.

[21] J. F. Molina, R. Gil, C. Bojacá, F. Gómez, and H. Franco, "Automatic detection of early blight infection on tomato crops using a color based classification strategy," in Proc. of 2014 XIX Symposium on Image, Signal Processing and Artificial Vision, pp. 1-5, 2014. Article (CrossRef Link)

[22] Y. Gu, S. Yoo, C. Park, Y. Kim, S. Park, J. Kim, and J. Lim, "BLITE-SVR: New forecasting model for late blight on potato using support-vector regression," Computers and Electronics in Agriculture, vol. 130, pp. 169-176, 2016. Article (CrossRef Link)

[23] G. Marcus, "Deep learning: A critical appraisal," arXiv preprint arXiv:1801.00631, 2018.

[24] D. Hughes and M. Salathé, "An open access repository of images on plant health to enable the development of mobile disease diagnostics," arXiv preprint arXiv:1511.08060, 2015.

[25] B. Zhang, L. Zhang, D. Zhang, and L. Shen, "Directional binary code with application to PolyU near-infrared face database," Pattern Recognition Letters, vol. 31, no. 14, pp. 2337-2344, 2010. Article (CrossRef Link)

[26] Y. Lu, S. Yi, N. Zeng, Y. Liu, and Y. Zhang, "Identification of rice diseases using deep convolutional neural networks," Neurocomputing, vol. 267, pp. 378-384, 2017. Article (CrossRef Link)

[27] W.-z. Liang, K. R. Kirk, and J. K. Greene, "Estimation of soybean leaf area, edge, and defoliation using color image analysis," Computers and electronics in agriculture, vol. 150, pp. 41-51, 2018. Article (CrossRef Link)

[28] M. M. Kamal, A. N. I. Masazhar, and F. A. Rahman, "Classification of Leaf Disease from Image Processing Technique," Indonesian Journal of Electrical Engineering and Computer Science, vol. 10, no. 1, pp. 191-200, 2018. Article (CrossRef Link)

[29] D. S. Tan, R. N. Leong, A. F. Laguna, C. A. Ngo, A. Lao, D. M. Amalin, and D. G. Alvindia, "AuToDiDAC: Automated tool for disease detection and assessment for cacao black pod rot," Crop Protection, vol. 103, pp. 98-102, 2018. Article (CrossRef Link)

[30] M. Sharif, M. A. Khan, Z. Iqbal, M. F. Azam, M. I. U. Lali, and M. Y. Javed, "Detection and classification of citrus diseases in agriculture based on optimized weighted segmentation and feature selection," Computers and electronics in agriculture, vol. 150, pp. 220-234, 2018. Article (CrossRef Link)

[31] K. Singh, S. Kumar, and P. Kaur, "Support vector machine classifier based detection of fungal rust disease in Pea Plant (Pisam sativam)," International Journal of Information Technology, vol. 11, no. 3, pp. 485-492, 2019. Article (CrossRef Link)

[32] R. Cristin, B. S. Kumar, C. Priya, and K. Karthick, "Deep neural network based Rider-Cuckoo Search Algorithm for plant disease detection," Artificial Intelligence Review, pp. 1-26, 2020. Article (CrossRef Link)

[33] A. Rath and J. Meher, "Disease detection in infected plant leaf by computational method," Archives of Phytopathology and Plant Protection, vol. 52, no. 19-20, pp. 1348-1358, 2019.

Article (CrossRef Link) 

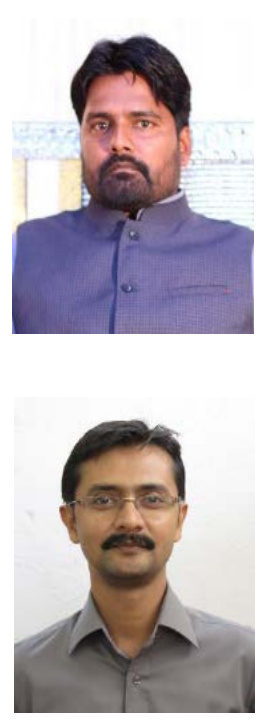

Syed Adnan received his MS degree in Computer Engineering from CASE (Center for Advanced Studies in Engineering) Islamabad, Pakistan in 2010, and the Ph.D. degree in Computer Engineering from University of Engineering and Technology Taxila, Pakistan in 2014. He has more than 15 years Research and Teaching experience. Currently, he is serving as Assistant Professor in Department of Computer Science at UET Taxila, Pakistan. His areas of interest are Acoustic Scene Analysis, Multimedia Signal Processing, Computer Vision and Machine Learning.

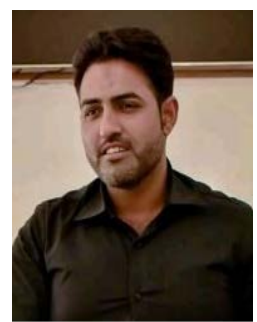

Aun Irtaza has completed his PhD in 2016 from FAST-NU, Islamabad Pakistan. During his $\mathrm{PhD}$ he remained working as a research scientist in the Gwangju Institute of Science and Technology (GIST), South Korea. He became an Associate Professor in 2017 and Department of Computer Science chair in 2018 in the University of Engineering and Technology (UET) Taxila, Pakistan. He is currently working as visiting Associate Professor in the University of Michigan-Dearborn. His research areas include computer vision, multimedia forensics, audio-signal processing, medical image processing, and Big data analytics. He has more than 40 publications in IEEE, Springer, and Elsevier Journals. 